\title{
Renal Venous Pattern: A New Parameter for Predicting Prognosis in Heart Failure Outpatients
}

\author{
Agata Puzzovivo 1@, Francesco Monitillo ${ }^{2}$, Pietro Guida ${ }^{3}$, Marta Leone ${ }^{4}$, Caterina Rizzo ${ }^{3}$, \\ Dario Grande $^{5}$ (D), Marco Matteo Ciccone ${ }^{5}$ and Massimo Iacoviello ${ }^{6, *(D)}$ \\ 1 Cardiology Unit, IRCCS istituto tumori “Giovanni Paolo II" di Bari, Italia; agatapuzzovivo@yahoo.it \\ 2 Emergency Cardiology Unit, University Policlinic Hospital, 70124 Bari, Italy; fmonitillo@libero.it \\ 3 Cardiovascular Department, Scientific Clinical Institutes Maugeri, Institute of Cassano delle Murge, \\ 70124 Bari, Italy; pieroguida@libero.it (P.G.); k.rizzo@live.it (C.R.) \\ 4 Cardiology Unit, SS Annunziata Hospital, 74123 Taranto, Italy; martaleo84@yahoo.it \\ 5 School of Cardiology, University of Bari, 70124 Bari, Italy; dario.grande@ymail.com (D.G.); \\ marcomatteo.ciccone@uniba.it (M.M.C.) \\ 6 University Cardiology Unit, Cardiothoracic Department, Policlinic University Hospital, \\ Piazza Giulio Cesare 11, 70124 Bari, Italy \\ * Correspondence: massimo.iacoviello@policlinico.ba.it; Tel.: +39-080-547-8622; Fax: +39-080-547-8796
}

Received: 21 August 2018; Accepted: 30 October 2018; Published: 3 November 2018

\begin{abstract}
Aim of the study: In chronic heart failure (CHF) patients, renal congestion plays a key role in determining the progression of renal dysfunction and a worse prognosis. The aim of this study was to define the role of Doppler venous patterns reflecting renal congestion that predict heart failure progression. Methods: We enrolled outpatients affected by CHF, in stable clinical conditions and in conventional therapy. All patients underwent a clinical evaluation, routine chemistry, an echocardiogram and a renal echo-Doppler. Pulsed Doppler flow recording was performed at the level of interlobular renal right veins in the tele-expiratory phase. The venous flow patterns were divided into five groups according to the fluctuations of the flow. Type A and B were characterized by a continuous flow, whereas type $C$ was characterized by a short interruption or reversal flow during the end-diastolic or protosystolic phase. Type $\mathrm{D}$ and $\mathrm{E}$ were characterized by a wide interruption and/or reversal flow. The occurrence of death and/or of heart transplantation and/or of hospitalization due to heart failure worsening was considered an event during follow-up. Results: During a median follow-up of 38 months, 126 patients experienced the considered end-point. Venous pattern C (HR 4.04; 95\% CI: 2.14-7.65; $p<0.001$ ), pattern D (HR 7.16; 95\% CI: 3.69-13.9; $p<0.001)$ and pattern E (HR 8.94; 95\% CI: 4.65-17.2; $p<0.001)$ were all associated with events using an univariate Cox regression analysis. Moreover, both the presence of pattern C (HR: 1.79; 95\% CI: $1.09-2.97 ; p$ : 0 ) and of pattern D or E (HR: 1.90; 95\% CI: 1.16-3.12; $p$ : 0.011) remained significantly associated to events using a multivariate Cox regression analysis after correction for a reference model with an improvement of the overall net reclassification index $(0.46 ; 95 \% \mathrm{CI}$ 0.24-0.68; $p<0.001)$. Conclusions: Our findings demonstrate the independent and incremental role of Doppler venous patterns reflecting renal congestion in predicting HF progression among CHF patients, thus suggesting its possible utility in daily clinical practice to better characterize patients with cardio-renal syndrome.
\end{abstract}

Keywords: cardiorenal syndrome; prognosis; heart failure; renal Doppler; congestion

\section{Introduction}

Over the last few decades, there has been a growing interest in the role of renal impairment in determining a worse prognosis of patients affected by chronic heart failure (CHF) [1-3]. In this setting 
it has been suggested that renal Doppler ultrasonography could have a role in detecting renal flow impairment and to better characterize renal function [4-7].

Using this technique, it is possible to evaluate not only the arterial flow and the arterial renal resistance, but also the renal venous blood flow [8-11]. In a population of outpatients and hospitalized heart failure patients, it has been recently demonstrated that the presence of an intra-renal intermittent venous flow with a biphasic or monophasic pattern [10] is associated with high central venous pressure, renal congestion and a worse prognosis. However, in this study, no data has been reported regarding the pattern observed in pre-eclamptic patients in which a reversal flow correspondent to right atrial contraction has been observed. This pattern seems to be between those showing a continuous and intermittent monophasic flow and is associated with an increased renal venous impedance index [11].

The aim of this study was to better define the role of all the possible renal Doppler venous patterns in predicting a worse prognosis in a large group of $\mathrm{CHF}$ outpatients in stable clinical conditions.

\section{Methods}

A population of consecutive outpatients affected by CHF of any origin and referred to the Heart Failure Unit of the Cardiology Unit of Policlinic Hospital of Bari were enrolled between 2013 and 2017. They were clinically stable for at least 30 days and had been receiving conventional medical therapy for at least 3 months if not contraindicated. Patients with acute worsening of kidney function or renal failure requiring dialysis were excluded from the study. All subjects gave their informed consent for inclusion before they participated in the study. The study was conducted in accordance with the Declaration of Helsinki, and the protocol was approved by the Ethics Committee of the Policlinic University Hospital of Bari (Project identification code: 4282).

\subsection{Baseline Evaluations}

At the time of enrollment a documented record of ischemic heart disease, arterial hypertension and diabetes mellitus was made for each patient. New York Heart Association (NYHA) class and arterial pressure were also evaluated. Heart rhythm and heart rate were assessed using 12-lead ECG.

Through echocardiographic evaluation (Vivid 7, GE Vingmed Ultrasound, General Electric, Milwaukee, WI, USA), the left ventricular ejection fraction (LVEF) was calculated using Simpson's rule. $\mathrm{E} / \mathrm{e}^{\prime}$ was calculated as the ratio between the early peak of $\mathrm{E}$ wave (E) at mitral pulsed Doppler and the mean of early peak of septal and lateral mitral annulus at tissue Doppler imaging ( $\left.e^{\prime}\right)$. Right ventricular systolic function was evaluated by calculating the systolic peak of tricuspid annulus excursion (TAPSE). Mitral (MR) and tricuspid regurgitation (TR) were semi quantitatively evaluated with arbitrary units ranging from 0 to 4 .

Dilatation of the inferior vena cava and its collapsibility during inspiration were evaluated in order to assess central venous pressure (CVP) [12]. If inferior cava vein diameter was $<2.1 \mathrm{~cm}$ and it collapsed $>50 \%$ with a sniff, a CVP of $3 \mathrm{~mm} \mathrm{Hg}$ (range, $0-5 \mathrm{~mm} \mathrm{Hg}$ ) was estimated. In presence of an inferior cava vein $>2.1 \mathrm{~cm}$ which collapsed $<50 \%$ with a sniff, a CVP of $15 \mathrm{~mm} \mathrm{Hg}$ (range, $10-20 \mathrm{~mm}$ $\mathrm{Hg}$ ) was considered. In presence of an inferior cava vein $<2.1 \mathrm{~cm}$, but with a collapse $<50 \%$, CVP was estimated as an intermediate value of $8 \mathrm{~mm} \mathrm{Hg}$ (range, $5-10 \mathrm{~mm} \mathrm{Hg}$ ).

Renal Doppler was performed after echocardiographic examination using the same echocardiograph and probe (4 MHz). The patient was moved into a sitting position and a posterior approach to the kidney was used. In order to obtain the renal arterial resistance index (RRI), the course of right or left kidney segmental arteries were visualized by color Doppler flow. Then at the middle tract level of the best one visualized, a pulsed Doppler was performed. Every effort was made in order to reach the best alignment of the ultrasonic beam. An average of 2 to 3 measurements of peak systolic velocity and end diastolic velocity were used to calculate RRI according to Peurcelot's formula, i.e., $100 \times[1-($ end diastolic velocity/peak systolic velocity) $][6,13]$. Venous Doppler was recorded at end-expiration. The analysis of venous patterns was performed by two observers (A.P., F.M.) who were blinded from patients' follow-up. We decided to classify the patterns based on previous papers [8-10] 
but propose a new type of classification. As shown in Figure 1, the following venous patterns were identified for each patient: Pattern A including flow patterns with normal velocity decrease of presystolic flow and biphasic pattern without interruption of telediastolic flow; Pattern B including patterns of continuous flow with minimal fluctuations; Pattern $C$ including patterns showing a short telediastolic interruption of forward flow or a short telediastolic reversal flow; Pattern D including patterns characterized by a biphasic interruption or reversal flow during the same cardiac cycle; Pattern E characterized by one forward and one reversal wave flow, i.e., monophasic intermittent pattern.

Blood samples were taken in order to assess serum creatinine, hemoglobin and NT-proBNP. The GFR was calculated using the Chronic Kidney Disease Epidemiology Collaboration equation (GFRCKD-EPI) [14]. By urine sample, normalbuminuria, microalbuminuria and macroalbuminuria were defined as the urinary albumin/creatinine ratio (UACR) of $<30,30$ to 299 , and $\geq 300 \mathrm{mg} / \mathrm{g}$.

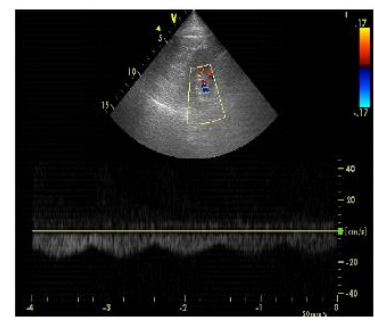

Type A

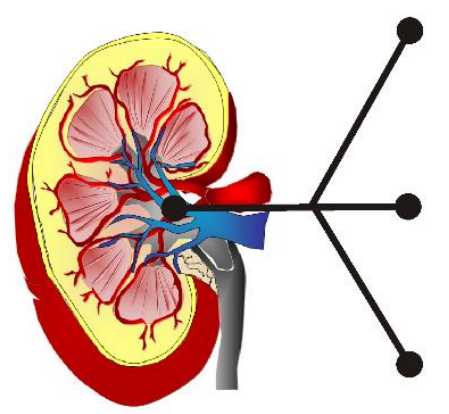

Type E

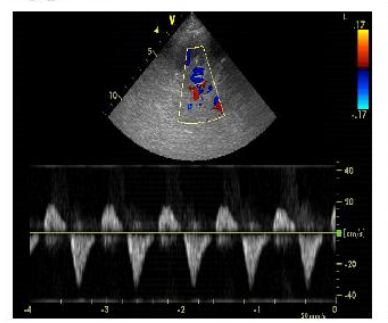

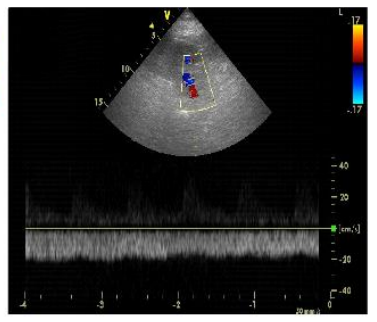

Type B

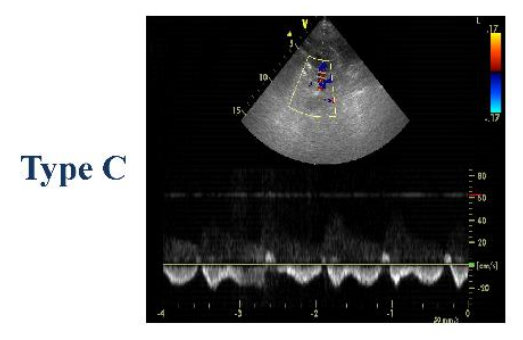

Type D

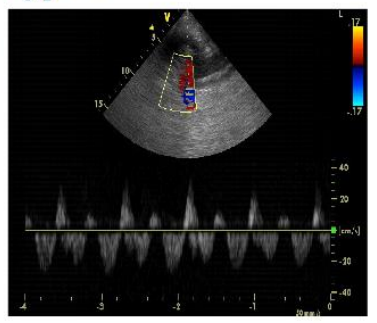

Continuous renal venous flow patterns

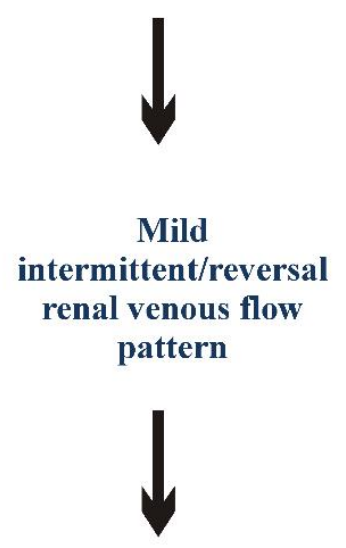

Intermittent/reversal renal venous flow patterns

Figure 1. The different detectable renal venous patterns are presented. Pattern A was a flow pattern with normal velocity decrease of presystolic flow and biphasic pattern without interruption of telediastolic flow; Pattern B a continuous flow pattern with minimal fluctuations; Pattern C a pattern showing a short telediastolic interruption of forward flow or a short telediastolic reversal flow; Pattern D a flow characterized by a biphasic interruption or reversal flow during the same cardiac cycle; Pattern E a flow characterized by one forward and one reversal wave flow, i.e., monophasic intermittent pattern.

\subsection{Follow-Up}

The patients received follow-up as outpatients according to the protocol of our Heart Failure Unit. Information about deaths and hospitalizations was collected to reflect on the underlying cause. The primary end-point was considered as the occurrence of death and/or heart transplantation due to heart failure worsening and/or unplanned hospitalizations due to acute decompensated heart failure (ADHF). The timing to the first event was considered for the analysis. 


\subsection{Statistical Analysis}

Continuous variables are expressed as mean values \pm SD. In 20 randomly selected patients, the renal venous pattern was evaluated twice by the first observer and then by a second observer. The second observer was blinded to the evaluation of the first observer. Intra- and interobserver reproducibility was evaluated as a percentage of concordance of pattern definition.

Categorical variables are expressed as absolute frequency or percentage. Comparisons among groups were performed using an ANOVA analysis whilst Newman-Keuls tests were used for post-hoc comparisons. Comparisons among groups of the categorical variables were performed with a chi-square test or Fisher test.

The event-free survival curves were based on Kaplan-Meier analyses. Univariate Cox's proportional hazards model was used to assess the association of variables with the endpoints. The reference model for the primary endpoint was developed with a Cox multivariate regression analysis. This included all univariate predictors by using a forward stepwise selection approach with $p$ $<0.05$ to retain covariates in the model. To avoid multicollinearity, redundant variables were dropped from the multivariable regression models. In the case of pairwise correlations between continuous variables exceeding 0.50 in Pearson's coefficient, the variable with the strongest individual effect size was included [15]. Finally, to test the independent association of venous Doppler renal variables with the primary endpoint, they were analyzed in a Cox multivariate regression analysis with all the variables of the reference model. The effect of adding the renal venous pattern to the reference model on risk classification was evaluated with the use of category-free net reclassification improvement (NRI) [16]. The index quantifies improvement in the prediction of a risk factor added to a reference model by evaluating any upward or downward movement in predicted risks. A valuable new risk factor tends to increase predicted risks for events and decrease risks for nonevents.

Finally, in order to demonstrate that the renal venous Doppler parameters were associated with the events independently, a multivariate Cox regression model including microalbuminuria, GFR-EPI, RRI and renal venous patterns was analyzed.

The analyses were carried out using Statistica software, version 6.1 (StatSoft Inc., Tulsa, Oklahoma) and STATA software, Version 12 (StataCorp, College Station, TX, USA). $p$-values of $<0.05$ were considered statistically significant.

\section{Results}

Out of 343 patients, a total of 338 patients were enrolled. Two patients were excluded because their RRIs were not valuable and another three patients were in dialytic treatment. The remaining patients constituted our study population whose baseline clinical characteristics are shown in Table 1 . Renal venous pattern was available in all the patients. All patients were in conventional therapy, $301(89 \%)$ were previously implanted with a cardioverter defibrillator and $112(33 \%)$ received cardiac resynchronization therapy. Sixty-one (18\%) patients were in atrial fibrillation.

\subsection{Reproducibility of Renal Venous Flow Pattern}

The intra-operator concordance in the classification of renal venous flow pattern was $95 \%$, whereas the interoperator concordance was $90 \%$.

\subsection{Clinical Correlates and Determinants of Renal Venous Flow Pattern}

Table 1 shows the characteristics of patients according to the renal venous pattern. The major observed differences concern the patients of group D and E, which had a greater functional limitation, lower values of blood pressure, worse renal function, higher values of NT-proBNP, mitral and tricuspid regurgitation, central venous pressure, higher mean values of RRI and lower values of hemoglobin, LVEF, and TAPSE. Group D and E patients also showed a lower percentage of ACE inhibitors or ARBs therapy and a higher mean dose of furosemide. Compared to patients with pattern A or B, 
patients with pattern $C$, were characterized by greater values of NT-proBNP and right ventricular pressures. Interestingly, in presence of type D and E, 32 and 30\% of patients, respectively, did not met echocardiographic criteria for high central venous pressure. In type $C$, this percentage was $70 \%$.

Table 1. Baseline clinical characteristics of all patients and of patients divided according to the venous pattern.

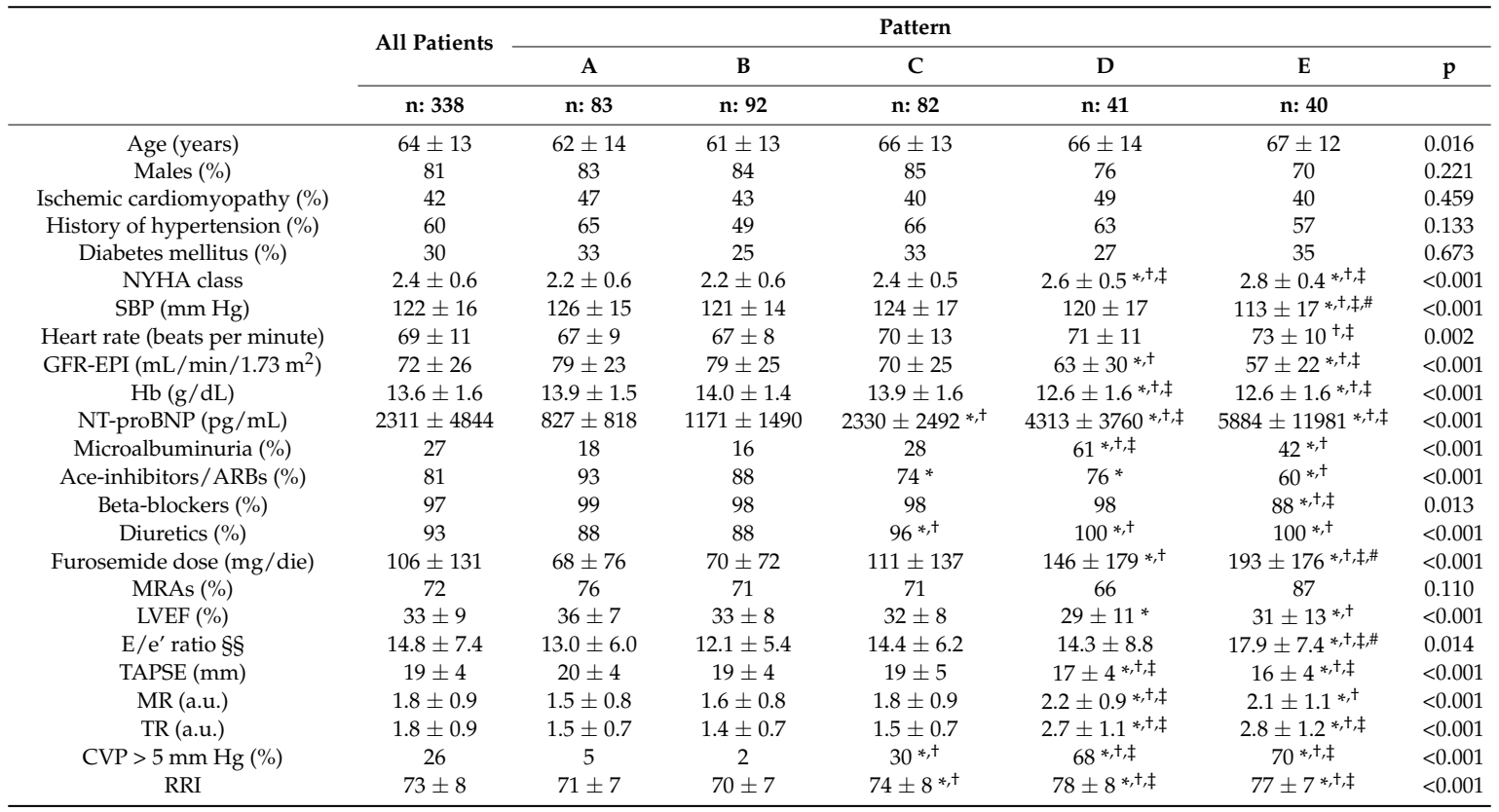

${ }^{*} p<0.05$ vs. pattern $\mathrm{A} ;{ }^{\dagger}$ vs. pattern B; ${ }^{\ddagger}$ vs. Pattern C; ${ }^{\#}$ vs. Pattern D at Newman-Keuls post-hoc analysis or Fisher test as appropriate. § Analysis for 307 patients without macroalbuminuria. $\S \S$ Available in 261 patients. Mean values \pm SD or Percentage of patients. The table shoes ANOVA test for continuous variables F-Fisher tests for categorical variables. For NT-proBNP analyses were performed after logarithmic transformation. ARBs: Angiotensin II receptor blockers; a.u., arbitrary units; CVP, central venous pressure; GFR-EPI, glomerular filtration rate by EPI formula; MRA: Aldosteron receptor antagonist; LVEF, left ventricular ejection fraction; MR, mitral regurgitation; NYHA, New York Heart Association; NT-proBNP: brain natriuretic peptide. RRI, renal resistance index; TAPSE, peak of tricuspid annular plane systolic excursion; TR, tricuspid regurgitation.

\subsection{Renal Venous Flow Pattern and Prognosis}

During a mean follow-up of $35 \pm 16$ months (median 38 months, interquartile range $16-48$ months), 78 patients died: 8 from sudden cardiac death, 54 because of worsening HF, 24 from other causes (6 due to non-cardiovascular causes). One hundred and twenty-two patients experienced at least one hospitalization due to ADHF and twenty-three underwent heart transplantation and/or left ventricular assist device. A total number of 126 patients experienced heart failure progression events.

Throughout univariate analysis, venous pattern C (HR 4.04; 95\% CI: 2.14-7.65; $p<0.001$ ), pattern D (HR 7.16; 95\% CI: 3.69-13.9; $p<0.001$ ) and pattern E (HR 8.94; 95\% CI: 4.65-17.2; $p<0.001$ ) showed a significantly greater probability of primary point events in comparison with Pattern A which was the reference group. No differences were found between pattern $A$ and $B$ and between pattern $\mathrm{E}$ and $\mathrm{D}$, whereas both these pattern showed a greater probability of events when compared with pattern C. For the other analyses, we categorized three subgroups of patients: those with a continuous renal venous flow pattern (CRVP, i.e., patients showing pattern $A$ and $B$ ), those with a mild intermittent/reversal renal venous flow pattern (MIRVP, i.e., patients showing pattern C) and those with an intermittent/reversal renal venous flow pattern (IRVP. i.e., patients showing pattern D and E). Table 2 shows the univariate predictors of events. 
Table 2. Univariate analysis for primary end-point. For abbreviations see Table 1.

\begin{tabular}{ccc}
\hline & \multicolumn{2}{c}{ Cox Univariate Analysis } \\
\cline { 2 - 3 } & HR (95\% CI) & $p$ \\
\hline Age & $1.02(1.01-1.04)$ & 0.001 \\
Diabetes & $1.47(1.03-2.12)$ & 0.036 \\
Ischemic aetiology & $1.52(1.07-2.15)$ & 0.019 \\
Systolic arterial pressure & $0.98(0.96-0.99)$ & $<0.001$ \\
ACE-I and/or ARBs & $0.33(0.23-0.49)$ & $<0.001$ \\
Beta-blocker & $0.28(0.14-0.57)$ & $<0.001$ \\
NYHA class III & $5.67(3.82-8.39)$ & $<0.001$ \\
Heart rate $>$ 70 & $1.64(1.15-2.35)$ & 0.007 \\
LVEF $<30 \%$ & $3.27(2.58-4.75)$ & $<0.001$ \\
Mitral regurgitation & $1.94(1.62-2.33)$ & $<0.001$ \\
TAPSE < 15 & $2.91(2.03-4.16)$ & $<0.001$ \\
Tricuspid regurgitation & $1.87(1.59-2.21)$ & $<0.001$ \\
CVP $>5$ mm Hg & $3.23(2.26-4.64)$ & $<0.001$ \\
GFR-EPI & $0.98(0.97-0.98)$ & $<0.001$ \\
Micro- or macroalbuminuria & $3.11(2.19-4.43)$ & $<0.001$ \\
Hemoglobin & $0.74(0.66-0.83)$ & $<0.001$ \\
NT-proBNP $(\times 100)$ & $1.03(1.02-1.03)$ & $<0.001$ \\
RRI $>$ 75 & $2.99(2.07-4.33)$ & $<0.001$ \\
\hline
\end{tabular}

As shown in Table 3, in the stepwise forward Cox regression analysis, which included all the univariate predictors, NT-proBNP, NYHA class III, RRI > 75, heart rate $>70$, MR, TAPSE $\leq 15$ remained associated with the occurrence of primary end-point, but not age, diabetes, systolic arterial pressure, therapy with ACE-inhibitors/Angiotensin receptor blockers, therapy with beta-blockers, $\mathrm{LVEF}<30 \%$, tricuspid regurgitation, CVP $>5 \mathrm{~mm} \mathrm{Hg}$, GFR-EPI, presence of micro- or macroalbuminuria, hemoglobin.

In the multivariate Cox regression model including all the variables of the reference model both the presence of a MIRVP, i.e., Pattern C, and the presence of a IRVP, i.e., Pattern D or E, remained significantly associated with the events as well as RRI $>75$ (Table 3). Moreover, the addition to the model including these parameters of MIRVP and IRVP improved reclassification according to NRI $(0.46 ; 95 \%$ CI $0.24-0.68 ; p<0.001)$.

Table 3. Reference model derived by using a forward stepwise Cox analysis where renal venous patterns categories have been included.

\begin{tabular}{ccccc}
\hline & \multicolumn{2}{c}{ Reference Model } & \multicolumn{2}{c}{$\begin{array}{c}\text { Reference Model Plus Renal } \\
\text { Venous Patterns }\end{array}$} \\
\cline { 2 - 5 } & HR (95\% CI) & $p$ & HR (95\% CI) & $p$ \\
\hline NYHA class III & $3.01(1.97-4.59)$ & $<0.001$ & $2.72(1.76-4.20)$ & $<0.001$ \\
Heart rate $>$ 70 & $1.69(1.17-2.44)$ & 0.005 & $1.55(1.07-2.27)$ & 0.005 \\
Mitral regurgitation & $1.29(1.07-1.56)$ & 0.008 & $1.25(1.04-1.52)$ & 0.008 \\
TAPSE $<$ 15 & $1.59(1.09-2.32)$ & 0.017 & $1.53(1.05-2.25)$ & 0.017 \\
NT-proBNP $(\times 100)$ & $1.020(1.015-1.026)$ & $<0.001$ & $1.02(1.012-1.023)$ & $<0.001$ \\
RRI $>$ 75 & $1.92(1.31-2.81)$ & 0.001 & $1.69(1.14-2.50)$ & 0.001 \\
CRVP (type A or B) & & & 1.00 & - \\
MIRVP (type C) & & & $1.79(1.09-2.97)$ & 0.023 \\
IRVP (type D or E) & & & $1.90(1.16-3.12)$ & 0.011 \\
\hline
\end{tabular}

CRVP: continuous renal venous flow pattern. IRVP: intermittent/reversal renal venous flow pattern. MIRVP: mild intermittent/reversal renal venous flow pattern. For the other abbreviations see Table 1.

When the presence of Pattern C and of Pattern D or E were added in a multivariate regression model including the other variables related with renal function, i.e., RRI, GFR-EPI and presence of 
micro- or macroalbuminuria, both remained significantly associated with events (HR: $2.24 ; 95 \%$ CI: 1.43-3.51; $p<0.001$ and HR: 3.55; 95\% CI: 2.30-5.47; $p<0.001$, respectively).

Figure 2 shows Kaplan Meier curves for the occurrence of death/hospitalization due to heart failure worsening and for the occurrence of death for all causes in patients with CRVP, MIRVP and IRVP.

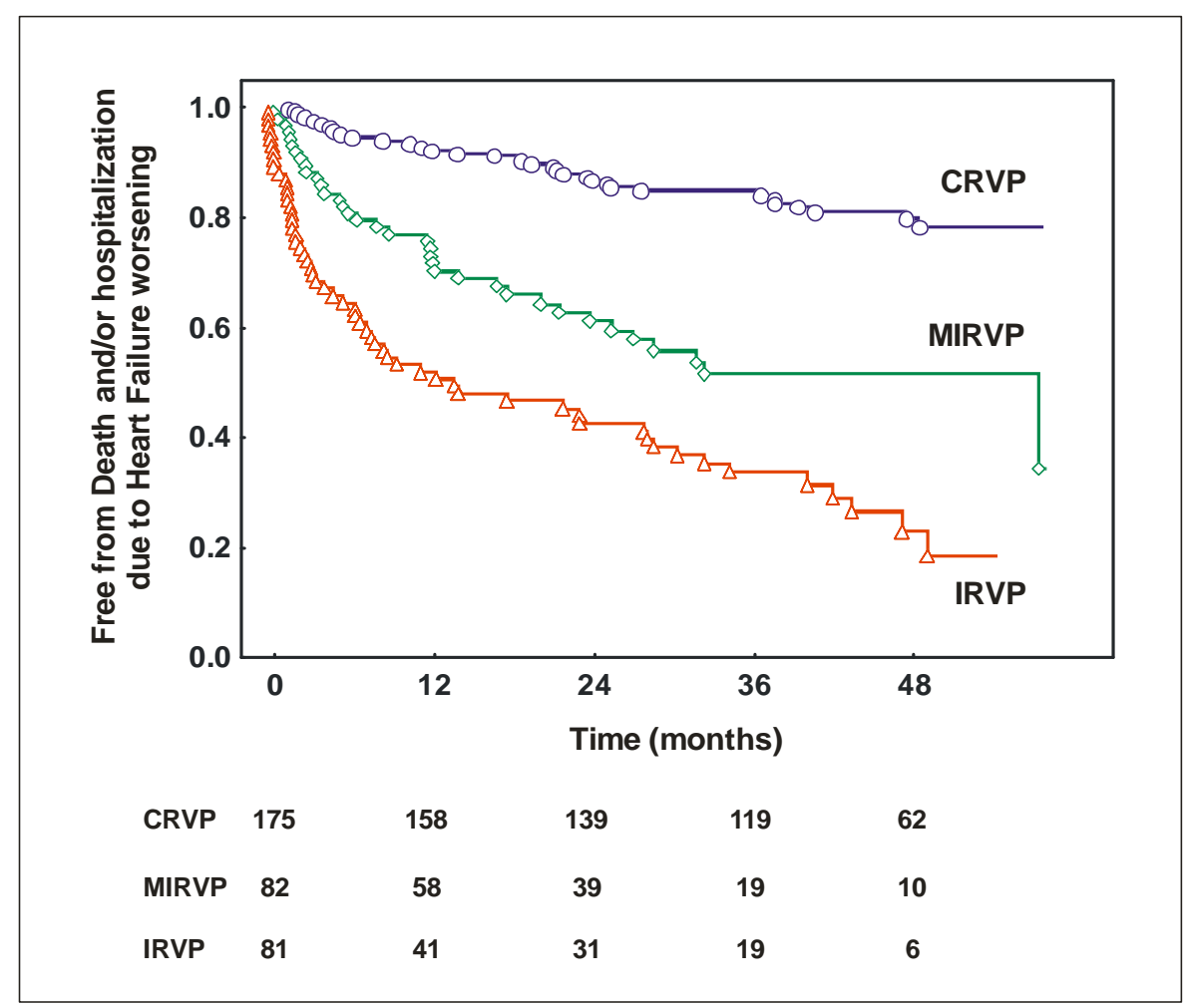

Figure 2. Kaplan Meier curves for the occurrence of death and/or hospitalization due to heart failure worsening in patients with continuous renal venous flow pattern (CRVP, i.e., Pattern A or B), mild intermittent/reversal renal venous flow pattern (MIRVP, i.e., Pattern C) and intermittent/reversal renal venous flow pattern (IRVP, i.e., Pattern D or E).

\section{Discussion}

The main finding of this study is that the renal Doppler venous patterns characterized by an intermittent or reversal flow show an independent and incremental role in predicting a worse prognosis of CHF outpatients. The predictive value of renal venous patterns seems to be related to its ability to better detect renal venous congestion. This strengthens the possible role of Doppler ultrasonography in daily clinical practice in order to better characterize the relationship between cardiac and renal dysfunction.

In CHF patients, renal function impairment and its worsening $[1-3,17]$ are frequently observed conditions which are independently associated with greater morbidity and mortality. This prognostic impact is due to the complex pathophysiological link that exists between the heart and the kidney. This has recently been defined as cardiorenal syndrome, i.e., the condition characterized by an acute or chronic dysfunction of one of the two organs which may induce the acute or chronic dysfunction of the other [2].

Among the pathophysiologic factors responsible for the progression of cardiorenal syndrome, the abnormalities in renal perfusion can play a key role [18]. An abnormal renal blood flow can be determined either by hypoperfusion related to low cardiac output or by congestion related to increased CVP [19]. The role of increased CVP in determining the progression of cardio-renal syndrome in both acute decompensated heart failure and chronic heart failure has been widely demonstrated $[19,20]$. High values of central venous pressure can be transmitted to the renal veins causing an increase in 
efferent arteriolar and glomerular capillary pressure, and consequently a reduction of the net filtration pressure and of the glomerular filtration rate [21].

In daily clinical practice, the estimation of CVP through the measurement of the inferior vena cava diameter [11] is the only echographic parameter that is routinely assessed and can be useful to detect the presence of renal congestion in heart failure patients. In this clinical setting the study of renal venous flow by the Doppler technique can improve the ability in detecting the presence of an increased renal venous pressure and, as a consequence, the presence of renal congestion. In presence of high right atrial pressures, the renal venous flow pulsatility can be altered and renal blood flow can be stopped or reversed. In a recent study by Iida et al. [10], the presence of an intermittent renal venous flow has been demonstrated to be strongly related with invasively measured high central venous pressure and it has been associated with a worse prognosis.

Our findings confirm these results but also offer further data supporting the possible usefulness of the study of renal venous flow in order to better detect renal congestion and to stratify prognosis of CHF outpatients. Like in the study of Iida and colleagues, in our series, the presence of intermittent venous patterns, that we defined as D or E, is associated with an increased estimated central venous pressure. However, it is worth noting that around $30 \%$ of patients showing these patterns do not present increased CVP assessed by inferior cava vein diameter. Moreover, the prognostic value of these patterns was independent and incremental from that of high CVP. As a consequence, these renal venous Doppler patterns seem to reflect more accurately than the estimated CVP of renal congestion.

Beyond the prognostic role of IRVP, i.e., patterns D and E, our data also demonstrate that another pattern, that we defined as MIRVP or pattern C, is independently associated with a worse prognosis. Although IRVP is poorly correlated with the presence of high CVP. This type of pattern has been previously described in pre-eclamptic patients [9] and it is characterized by an end-diastolic or proto-systolic interruption of the flow that can be associated to the presence of a reversal flow. Pattern $C$ can be the expression of a mild increase of central venous pressure, which becomes evident when a further increase of right atrial pressure is transmitted to renal veins and it is able to stop or reverse the blood flow. In particular, the right atrium pressure changes along the cardiac cycle which is responsible for the triphasic modulation of venous blood flow at the level of the inferior vena cava and hepatic veins. The transient increase of atrial pressure during atrial systole and/or the closure of tricuspid leaflets at the beginning of systole can cause an interrupted or a reversal flow. This hypothesis is supported by a recent study which evaluated the effect of volume expansion on intrarenal venous flow [11]. When the atrial pressures further increase, the interruption of the flow is longer and the intermittent patterns D and E can be observed. On the basis of these considerations, the presence of a mild intermittent/reversal renal flow pattern can detect early renal venous blood flow abnormalities related to congestion, thus also explaining its independent and incremental role in predicting a worse prognosis.

Although our findings about renal venous patters are similar to those recently published by lida and colleagues [10], there are several differences. First of all, we enrolled a larger series of patients who were in stable clinical conditions and who were followed-up over a longer period. Secondly, we identified the pattern $C$ as associated with a mild renal congestion and worse prognosis when compared with continuous renal flow patterns, i.e., A and B. Thirdly, we have demonstrated that the evaluation of venous flow pattern can integrate the information obtained from the assessment of RRI in order to identify patients more prone to experience worsening heart failure. Arterial and venous renal Doppler are able to offer different and complementary information. In fact, arterial Doppler and RRI can more narrowly reflect vascular and parenchymal renal abnormalities as well as neurohormonal hyperactivity and congestion related to high intrarenal pressures. Whereas renal venous patterns can more accurately reflect the presence of renal congestion. The independent association with the events of renal venous patterns and RRI supports this hypothesis. Finally, the relevant clinical role of arterial and venous Doppler ultrasonography is further supported by the fact that their prognostic value, 
in our series, is independent from the estimation of GFR and microalbuminuria which are the renal function parameters with a proven prognostic role in CHF outpatients [1,22].

Limitations of the study: This is an observational study which needs to be confirmed by larger multicenter studies. Although we demonstrated the prognostic relevance of renal venous Doppler pattern, GFR remains the main parameter for the assessment of renal function in type 2 cardiorenal syndrome. Renal venous Doppler pattern could provide a further characterization of renal function but it cannot be proposed in routine clinical practice. Further studies should confirm the independent prognostic value of renal venous patterns as well as evaluate if there is a relationship among changes in renal venous patterns, changes in the other parameters reflecting congestion and its relationship with a worse prognosis. This could be useful in order to understand if renal venous patterns can be proposed not only as marker of prognosis but also for tailoring patients' therapy in order to reduce the negative effects of renal congestion. Finally, although the evaluation of the renal venous pattern can be easily obtained at the end of a standard echocardiographic examination, future studies should also demonstrate the cost effectiveness of this examination in heart failure patients.

In conclusion, renal venous Doppler patterns reflecting renal congestion show an independent and incremental role in predicting a worse outcome in CHF outpatients. These results suggest the possible clinical usefulness of renal venous Doppler evaluations in better characterizing cardio-renal syndrome. Therefore, the present findings may represent the basis for further studies in larger samples aimed at defining the possible use of the renal venous Doppler in daily clinical practice, as well as the possibility of using it for a tailored therapeutic approach.

Author Contributions: A.P. conceived and designed the study, contributed to data collection, analysed and interpreted the data, drafted the article and critically reviewed its intellectual content, and finally approved the version to be submitted for publication. F.M. contributed to data collection, analysed and interpreted the data, drafted the article and critically reviewed its intellectual content, and finally approved the version to be submitted for publication. P.G. performed statistical analysis, interpreted the data, drafted the article and critically reviewed its intellectual content, and finally approved the version to be submitted for publication. M.L. contributed to data collection, critically reviewed the article and finally approved the version to be submitted for publication. C.R. contributed to data collection, reviewed the article's intellectual content, and finally approved the version to be to be submitted for publication. D.G. analysed and interpreted the data, drafted the article and critically reviewed its intellectual content, and finally approved the version to be submitted for publication. M.M.C. contributed towards designing the study, interpreting the data, critically reviewing the article's intellectual content, and finally approving the version to be submitted for publication. M.I. conceived and designed the study, analysed and interpreted the data, drafted the article and critically reviewed its intellectual content, and finally approved the version to be submitted for publication.

Acknowledgments: We thank Anna Cavallo and Caterina Giardino for their helpful contributions in the enrolment and management of the patients.

Conflicts of Interest: The authors declare no conflicts of interest.

\section{References}

1. Hillege, H.L.; Girbes, A.R.; de Kam, P.J.; Boomsma, F.; de Zeeuw, D.; Charlesworth, A.; Hampton, J.R.; van Veldhuisen, D.J. Renal function, neurohormonal activation, and survival in patients with chronic heart failure. Circulation 2000, 102, 203-210. [CrossRef] [PubMed]

2. Ronco, C.; Haapio, M.; House, A.A.; Anavekar, N.; Bellomo, R. Cardiorenal syndrome. J. Am. Coll. Cardiol. 2008, 52, 1527-1539. [CrossRef] [PubMed]

3. Damman, K.; Kalra, P.R.; Hillege, H. Pathophysiological mechanisms contributing to renal dysfunction in chronic heart failure. J. Renal Care 2010, 36, 18-26. [CrossRef] [PubMed]

4. Ennezat, P.V.; Maréchaux, S.; Six-Carpentier, M.; Pinçon, C.; Sediri, I.; Delsart, P.; Gras, M.; Mounier-Véhier, C.; Gautier, C.; Montaigne, D.; et al. Renal resistance index and its prognostic significance in patients with heart failure with preserved ejection fraction. Nephrol. Dial. Transplant. 2011, 26, 3908-3913. [CrossRef] [PubMed]

5. Doi, Y.; Iwashima, Y.; Yoshihara, F.; Kamide, K.; Hayashi, S.I.; Kubota, Y.; Nakamura, S.; Horio, T.; Kawano, Y. Renal Resistive Index and Cardiovascular and Renal Outcomes in Essential Hypertension. Hypertension 2012, 60, 770-777. [CrossRef] [PubMed] 
6. Ciccone, M.M.; Iacoviello, M.; Gesualdo, L.; Puzzovivo, A.; Antoncecchi, V.; Doronzo, A.; Monitillo, F.; Citarelli, G.; Paradies, V.; Favale, S. The renal arterial resistance index: A marker of renal function with an independent and incremental role in predicting heart failure progression. Eur. J. Heart Fail. 2014, 16, 210-216. [CrossRef] [PubMed]

7. Bigé, N.; Lévy, P.P.; Callard, P.; Faintuch, J.M.; Chigot, V.; Jousselin, V.; Ronco, P.; Boffa, J.J. Renal arterial resistive index is associated with severe histological changes and poor renal outcome during chronic kidney disease. BMC Nephrol. 2012, 13, 139. [CrossRef] [PubMed]

8. Jeong, S.H.; Jung, D.C.; Kim, S.H.; Kim, S.H. Renal venous doppler ultrasonography in normal subjects and patients with diabetic nephropathy: Value of venous impedance index measurements. J. Clin. Ultrasound 2011, 39, 512-518. [CrossRef] [PubMed]

9. Gyselaers, W.; Staelens, A.; Mesens, T.; Tomsin, K.; Oben, J.; Vonck, S.; Verresen, L.; Molenberghs, G. Maternal venous Doppler characteristics are abnormal in pre-eclampsia but not in gestational hypertension. Ultrasound Obstet. Gynecol. 2015, 45, 421-426. [CrossRef] [PubMed]

10. Iida, N.; Seo, Y.; Sai, S.; Machino-Ohtsuka, T.; Yamamoto, M.; Ishizu, T.; Kawakami, Y.; Aonuma, K. Clinical Implications of Intrarenal Hemodynamic Evaluation by Doppler Ultrasonography in Heart Failure. JACC Heart Fail. 2016, 4, 674-682. [CrossRef] [PubMed]

11. Nijst, P.; Martens, P.; Dupont, M.; Tang, W.H.W.; Mullens, W. Intrarenal Flow Alterations During Transition from Euvolemia to Intravascular Volume Expansion in Heart Failure Patients. JACC Heart Fail. 2017, 5, 672-681. [CrossRef] [PubMed]

12. Rudski, L.G.; Lai, W.W.; Afilalo, J.; Hua, L.; Handschumacher, M.D.; Chandrasekaran, K.; Solomon, S.D.; Louie, E.K.; Schiller, N.B. Guidelines for the echocardiographic assessment of the right heart in adults: A report from the American Society of Echocardiography endorsed by the European Association of Echocardiography, a registered branch of the European Society of Cardiology, and the Canadian Society of Echocardiography. J. Am. Soc. Echocardiogr. 2010, 23, 685-713. [PubMed]

13. Pourcelot, L. Velocimetrie ultrasonore Doppler Seminaire INSERM; Editions INSERM: Paris, France, 1974; pp. 213-240.

14. Levey, A.S.; Stevens, L.A.; Schmid, C.H.; Zhang, Y.L.; Castro, A.F., 3rd; Feldman, H.I.; Kusek, J.W.; Eggers, P.; Van Lente, F.; Greene, T.; et al. A new equation to estimate glomerular filtration rate. Ann. Intern. Med. 2009, 150, 604-612. [CrossRef] [PubMed]

15. Slinker, B.K.; Glantz, S.A. Multiple linear regression: Accounting for multiple simultaneous determinants of a continuous dependent variable. Circulation 2008, 117, 1732-1737. [CrossRef] [PubMed]

16. Pencina, M.J.; D'Agostino RBSr Steyerberg, E.W. Extensions of net reclassification improvement calculations to measure usefulness of new biomarkers. Stat. Med. 2011, 30, 11-21. [CrossRef] [PubMed]

17. Damman, K.; Jaarsma, T.; Voors, A.A.; Navis, G.; Hillege, H.L.; van Veldhuisen, D.J.; COACH Investigators. Both in- and out-hospital worsening of renal function predict outcome in patients with heart failure: Results from the Coordinating Study Evaluating Outcome of Advising and Counseling in Heart Failure (COACH). Eur. J. Heart Fail. 2009, 11, 847-854. [CrossRef] [PubMed]

18. Chade, A.R. Renal vascular structure and rarefaction. Compr. Physiol. 2013, 3, 817-831. [PubMed]

19. Damman, K.; Navis, G.; Smilde, T.D.; Voors, A.A.; van der Bij, W.; van Veldhuisen, D.J.; Hillege, H.L. Decreased cardiac output, venous congestion and the association with renal impairment in patients with cardiac dysfunction. Eur. J. Heart Fail. 2007, 9, 872-878. [CrossRef] [PubMed]

20. Iacoviello, M.; Puzzovivo, A.; Monitillo, F.; Saulle, D.; Lattarulo, M.S.; Guida, P.; Forleo, C.; Gesualdo, L.; Favale, S. Independent role of high central venous pressure in predicting worsening of renal function in chronic heart failure outpatients. Int. J. Cardiol. 2013, 162, 261-263. [CrossRef] [PubMed]

21. Jessup, M.; Costanzo, M.R. The cardiorenal syndrome: Do we need a change of strategy or a change of tactics? J. Am. Coll. Cardiol. 2009, 53, 597-599. [CrossRef] [PubMed]

22. Jackson, C.E.; Solomon, S.D.; Gerstein, H.C.; Zetterstrand, S.; Olofsson, B.; Michelson, E.L.; Granger, C.B.; Swedberg, K.; Pfeffer, M.A.; Yusuf, S.; et al. Albuminuria in chronic heart failure: Prevalence and prognostic importance. Lancet 2009, 374, 543-550. [CrossRef]

(C) 2018 by the authors. Licensee MDPI, Basel, Switzerland. This article is an open access article distributed under the terms and conditions of the Creative Commons Attribution (CC BY) license (http:/ / creativecommons.org/licenses/by/4.0/). 